\title{
A Brief Review on Bioinspired ZnO Superhydrophobic Surfaces: Theory, Synthesis, and Applications
}

\author{
Jun Wu, Jing Chen, Jun Xia, Wei Lei, and Bao-ping Wang \\ Electronic Science \& Engineering, Southeast University, Nanjing 210096, China \\ Correspondence should be addressed to Jun Wu; discover_wj626@126.com and Jun Xia; xiajun@seu.edu.cn
}

Received 22 May 2013; Revised 18 October 2013; Accepted 18 October 2013

Academic Editor: Manish U. Chhowalla

Copyright (C) 2013 Jun Wu et al. This is an open access article distributed under the Creative Commons Attribution License, which permits unrestricted use, distribution, and reproduction in any medium, provided the original work is properly cited.

\begin{abstract}
Due to the intensive demand in the development of superhydrophobic surfaces both in theory and application, superhydrophobic research on $\mathrm{ZnO}$ materials has exponentially grown over the last several years. One of the main advantages of the $\mathrm{ZnO}$ materials is the relative easiness to produce various surface morphologies, which is quite meaningful to study the influence of morphology on wetting property. The synthesis approaches of $\mathrm{ZnO}$ materials include thermal oxidization, hydrothermal method, chemical etching, spray coating technique, electrochemical method, and others. This review is a representation of the recent achievement on $\mathrm{ZnO}$ superhydrophobic surfaces.
\end{abstract}

\section{Introduction}

When a liquid drop is in contact with a solid surface, two distinct regimes of liquid spreading are observed depending on the spreading factor $S\left(S=\gamma_{\mathrm{sg}}-\gamma_{\mathrm{ls}}-\gamma\right)$, where $\gamma_{\mathrm{ls}}, \gamma_{\mathrm{sg}}$, and $\gamma$ are the interfacial tensions between liquid and solid, solid and gas, and gas and liquid [1]. The spreading factor $S$ indicates whether the formation of a liquid film is energetically favorable on a solid surface. A positive spreading factor $S$ means that the energy state with a liquid film on the solid surface is lower than that without a liquid film, implying that a liquid film will be preferably formed on the solid surface, that is, complete wetting. A negative spreading factor $S$, on the other hand, means a liquid drop will be formed on the solid surface with a certain contact angle, that is, partial wetting. In the partial wetting regime, if a contact angle is less than $90^{\circ}$, a surface is called hydrophilic. If a contact angle is larger than $90^{\circ}$, a surface is called hydrophobic. In particular, a surface with a contact angle larger than $150^{\circ}$ is considered to be superhydrophobic [2]. Therefore, the surface wetting property, that is, droplet contact angle, is closely related to the interfacial energies. However, as there exist irregular or regular patterns on the liquid and solid interface on superhydrophobic surfaces, droplet contact angle should be determined by overall considering interfacial energies and the structural parameters.
Meanwhile, superhydrophobic surfaces have kept attracting considerable interests due to the observation of various superhydrophobic phenomena in nature [2-13]. The most representative example of natural superhydrophobic surfaces is a lotus leaf [2]. On a lotus leaf, microscale bumps are covered with wax crystalloids. These surface structures render a liquid in contact with only the top of the surface structures, thereby resulting in the significant decrease of the adhesion energy between a liquid and the solid surface. This feature enables a lotus leaf to possess water repellency and selfcleaning properties. Then, several other examples of natural superhydrophobic surfaces have been discovered, including wings of butterfly [4], duck feathers [6], legs of the water strider [7, 8], integument of water walking arthropod [11], and covering of the desert beetle $[12,13]$. Different structural morphologies on these surfaces result in the superhydrophobicity, which helps to realize the decontamination, drag reduction, or even water collection. Therefore, both the scientific world and engineers show great interests in such special surfaces. In one case, it is highly desirable to make clear how the interfacial energy influences the wetting property on patterned surfaces. In another case, it is also quite important to design different structures to achieve desired superhydrophobicities.

The wetting property, that is, contact angle, can be calculated by considering the force balance at the three-phase 
contact line. Alternatively, the contact angle at which the thermodynamic energy becomes minimum can be sought. The detailed calculation methods will be introduced in the following section. Meanwhile, as various synthesis techniques to engineer superhydrophobic surfaces have already been developed and reviewed in several articles [14-17], for example, sol-gel reactions [18-20], electrochemical deposition [2123], layer-by-layer deposition [24-27], spin coating [28], plasma technology [29], and electrical spinning [30], and the materials utilized in these approaches vary from polymers, metals, and other inorganic materials to composites [31-38], we will not cover all these well-known experimental methods and materials in this brief review. Alternatively, we will focus on the recent advances in $\mathrm{ZnO}$ superhydrophobic materials, which feature a direct wide band gap $(3.37 \mathrm{eV})$ and large exciton binding energy ( $60 \mathrm{meV})$, which have been promising for optoelectronic devices and functional materials such as solar cells [39], light emitting diodes [40], sensors [41], and photodetector [42]. It is expected to explore multifunctional superhydrophobic surfaces based on $\mathrm{ZnO}$ [43-45].

In this review, therefore, the scope has been limited to the description of wetting property of superhydrophobic surfaces and also the recent advances in synthesis approaches of different $\mathrm{ZnO}$ superhydrophobic materials. There are three sections, including theory in wetting property, synthesis approaches, and conclusions.

\section{Theory}

2.1. Apparent Contact Angle. The contact angle is the manifestation of the interaction among interfacial tensions of the liquid-gas interface $\gamma$, liquid-solid interface $\gamma_{\mathrm{ls}}$, and solidgas interface $\gamma_{\text {sg }}$ and can be calculated by considering the force balance at the three-phase contact line. It is assumed that interfacial tensions along the horizontal direction at the three-phase contact line are at equilibrium, thereby resulting in the following Young relation (Figure 1(a)):

$$
\gamma_{\mathrm{ls}}+\gamma \cos \theta=\gamma_{\mathrm{sg}} \text {. }
$$

Alternatively, the contact angle at which the thermodynamic energy becomes minimum can also be sought. When a three-phase line makes a virtual displacement of $d x$ as shown in Figure 1(b), the accompanying change of thermodynamic energy is calculated in the following way [46]:

$$
d E=\gamma_{\mathrm{ls}} d x-\gamma_{\mathrm{sg}} d x+\gamma d x \cos \theta
$$

At thermodynamic energy equilibrium, $d E$ should be zero, and this condition leads to Young's relation as well.

The above analysis is based on the assumption that the solid has a flat surface. However, the thermodynamic approach can also be extended to a superhydrophobic surface as long as the length scale of the roughness is much smaller than the size of the liquid drop. On a rough surface, two distinct configurations of a liquid drop can be envisioned. First, it can be assumed that the liquid completely wets the rough surface while it moves by $d x$. Then, a liquid-solid interface will displace a solid-vapor interface by $r d x$ as shown in Figure 2(a) instead of $d x$ on a flat surface, where roughness $r$ is defined as the ratio of actual surface area versus projected surface area $(r>1)$. Then, the accompanying change of thermodynamic energy will be expressed as follows:

$$
d E=\gamma_{\mathrm{ls}} r d x-\gamma_{\mathrm{sg}} r d x+\gamma d x \cos \theta_{W}
$$

where $\theta_{W}$ is an apparent contact angle on a rough surface. The condition for thermodynamic energy minimum leads to the following Wenzel equation [47]:

$$
\cos \theta_{W}=\frac{r\left(\gamma_{\mathrm{sg}}-\gamma_{\mathrm{ls}}\right)}{\gamma}=r \cos \theta .
$$

As indicated by this equation, the Wenzel model predicts that roughness amplifies a surface wettability. That is, roughness makes a hydrophilic surface more hydrophilic and a hydrophobic surface more hydrophobic.

In another case, it can also be assumed that a liquid does not penetrate into the roughness, contacting only the top surfaces of the roughness. In this case, a composite interface of liquid-gas and liquid-solid between the overall liquid and solid interface is resultant as shown in Figure 2(b). Then, the corresponding energy change will be given by

$$
d E=\gamma_{\mathrm{ls}} \phi_{s} d x-\gamma_{\mathrm{sv}} \phi_{s} d x+\gamma\left(1-\phi_{s}\right) d x+\gamma d x \cos \theta_{C},
$$

where $\phi_{s}<1$ is an area fraction of liquid-solid interface and $\theta_{C}$ is an apparent contact angle on a rough surface. The condition for the minimization of the thermodynamic energy leads to the following Cassie-Baxter equation [48]:

$$
\cos \theta_{C}=\phi_{s} \cos \theta-\left(1-\phi_{s}\right) .
$$

Different from the Wenzel model, the Cassie-Baxter model predicts that a surface becomes more hydrophobic as the solid fraction gets smaller regardless of the intrinsic contact angle on the surface.

2.2. Contact Angle Hysteresis. On any real solid surface, people found that there exists a wide range of "metastable" contact angles when a liquid meniscus scans the solid surface. Because there are free energy barriers which exist between these metastable states, a true "equilibrium" contact angle is almost impossible to measure in real time. The previously mentioned Wenzel and Cassie theories are therefore only applicable in prediction of the thermodynamically stable contact angles in theory. Therefore, contact angle hysteresis defined as the difference of advancing angle and receding angle is usually measured to fully characterize any surface. A typical method to obtain an advancing contact angle is to gradually inject liquid into a sessile drop with a syringe and measure the contact angle when the liquid-solid-air contact line subsequently starts to move outward. Conversely, the method to obtain a receding contact angle is to gradually withdraw liquid out of a drop and measure the angle when the contact line starts to move inward.

In another case, tilting angle $\theta_{T}$ can be adopted to quantify this hysteresis effect. For example, when a liquid drop is placed on a titled plate as shown in Figure 3, a drop will not slip off until the following condition is satisfied [49]:

$$
m g \sin \theta_{T}=L \gamma\left(\cos \theta_{r}-\cos \theta_{a}\right),
$$




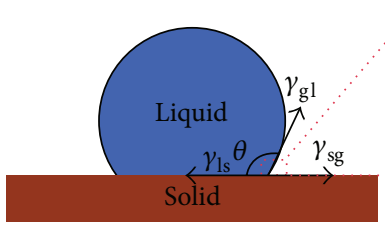

(a)

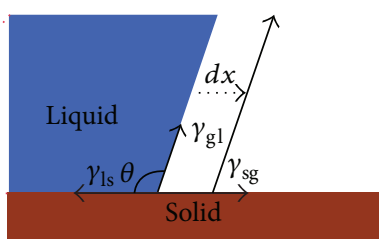

(b)

Figure 1: (a) A force balance along the horizontal direction at three-phase contact line is considered. (b) A contact angle at which thermodynamic energy becomes minimum can be derived when a liquid moves by $d x$.

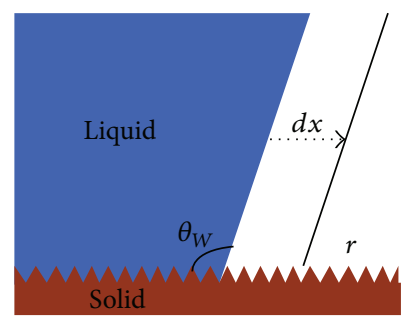

(a)

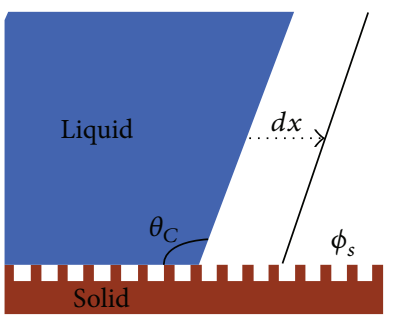

(b)

FIGURE 2: (a) Wenzel's model: a liquid drop homogeneously wets a solid surface. (b) Cassie-Baxter's model: a liquid drop only touches the top of a roughness on a solid surface.

where $L$ is a geometric factor that depends on the structure types, and determines the effective three-phase contact line length along the droplet boundary. The surface that has stronger pinning force will exhibit larger $\theta_{T}$ value, according to which the pinning force can be calculated.

As the apparent contact angle is modified on a rough surface from the intrinsic angle on a smooth surface, a contact angle hysteresis is also modified. While both the CassieBaxter model and Wenzel model predict the increase of contact angle on a rough hydrophobic surface, they predict opposite trends for a contact angle hysteresis. It is widely accepted that a nonwetted (i.e., Cassie-Baxter state) surface exhibits a decrease of contact angle hysteresis due to the reduced contact area between a liquid and a solid, and a wetted surface (i.e., Wenzel state) shows a significant increase due to the increased contact area between liquid and solid.

\section{ZnO Superhydrophobic Surfaces}

According to the theoretical analysis, the wetting states and the closely related wetting behavior vary with the surface structures and surface energies. Meanwhile, great progress has been achieved in the design and synthesis of bioinspired superhydrophobic surfaces based on different $\mathrm{ZnO}$ structures [50-78], among which most of them were modified with low surface energy materials, for example, silane and fluorination, to eliminate the influence of polarity of $\mathrm{ZnO}$ materials on wetting property. We reviewed the related literature published since the year of 2010. Here are the main synthesis methods introduced.

3.1. Thermal Oxidization. In microfabrication field, thermal oxidation is an effective way to produce a thin layer of oxide on the surface of a material. This technique forces an oxidizing agent to diffuse into the material at high temperature and react with it. The materials can be processed in this technique include silicon, metal, and others. By using thermal oxidization method, researchers got $\mathrm{ZnO}$ superhydrophobic surfaces $[50,51]$. Barshilia et al. deposited $\mathrm{ZnO}$ coatings with varying thicknesses (300-1800 nm) by sputtering a Zn target in $\mathrm{Ar}$ plasma followed by $1 \mathrm{~h}$ oxidation at $350^{\circ} \mathrm{C}$ in $\mathrm{O}_{2}$ environment [50]. $\mathrm{ZnO}$ coatings prepared under these conditions were further annealed for $2 \mathrm{~h}$ at $450^{\circ} \mathrm{C}$ in vacuum to improve the optical transparency. Except from the extraordinary water repellency (with contact angle $>155^{\circ}$ ) as indicated in Figure 4 , they even proved that $\mathrm{ZnO}$ superhydrophobic coating on the absorber surface has improved absorptance $(>0.96)$ and excellent broadband antireflection in the visible range of the solar spectrum. The multifunctional $\mathrm{ZnO}$ coating was stable up to $450^{\circ} \mathrm{C}$ (in air and vacuum), indicating its reliability for high temperature photothermal conversion applications.

3.2. Hydrothermal Method. Hydrothermal method is a useful tool to produce different chemical compounds and materials using closed-system physical and chemical processes flowing in aqueous solutions at high temperatures and certain pressures, and is also widely adopted to synthesize different $\mathrm{ZnO}$ micro/nanostructures [52-62]. Mondal et al. fabricated nanocolumnar thin film (Figure 5) under modified hydrothermal conditions [52]. The growth of $\mathrm{ZnO}$ nanocolumnar on glass surfaces takes place as a result of the hydrolysis of zinc sulfate by ethanolamine at $100^{\circ} \mathrm{C}$ without employing any templates or surfactants. The evolved superhydrophobic thin film becomes hydrophilic upon UV light exposure and the film reverts back to its original superhydrophobic character upon storage in the dark. Due 


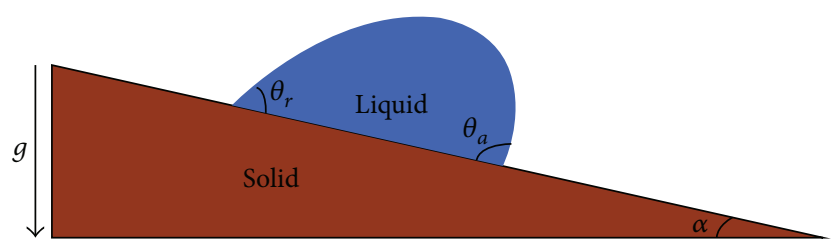

Figure 3: Liquid drop on the slanted solid plate. A liquid drop begins to slide down when the slant angle of the plate is larger than the threshold angle at which gravity balances with contact angle hysteresis.

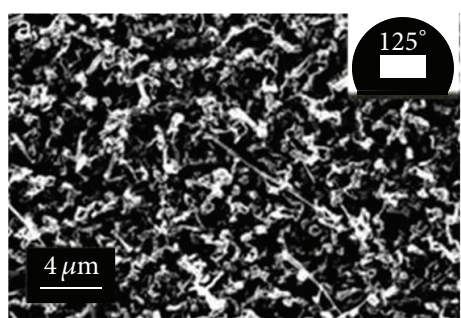

(a)

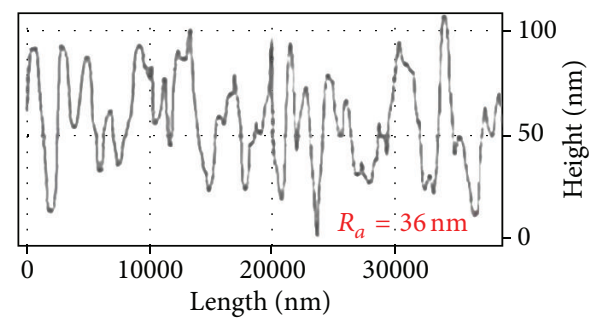

(b)

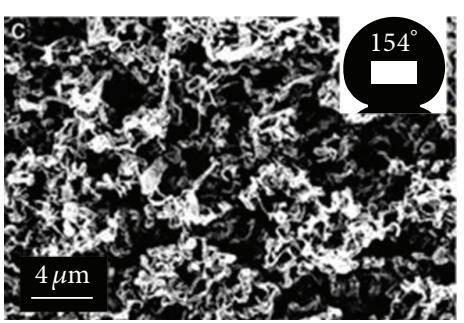

(c)

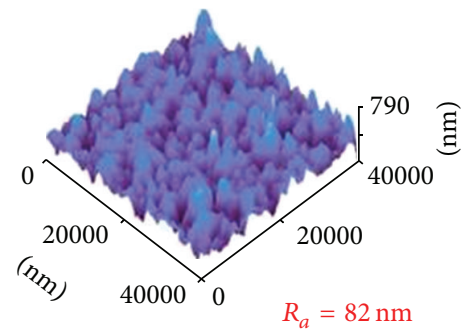

(d)

FIGURE 4: FESEM micrographs of $\mathrm{ZnO}$ nanoclustered film (a) with $433 \mathrm{~nm}$ thickness, and (b) corresponding AFM 2D roughness profile. (c) The FESEM image of $1300 \mathrm{~nm}$ optimized thickness with its (d) 3D morphology. The thickness of ZnO nanostructured coating was controlled by sputtering time. Copyright 2012, Elsevier.

to UV light illumination an electron-hole pair is generated in the $\mathrm{ZnO}$ thin film. Some of the holes react with lattice oxygen leading to oxygen vacancies in the film. The photogenerated electrons are captured by lattice zinc ions to form surface trapped electron sites. Hydroxyl adsorption on the photogenerated defect sites is kinetically favorable. This leads to the dissociative adsorption of water molecules at these sites, inducing hydrophilicity. However, on dark storage the superhydrophilic thin film recovers its original superhydrophobicity. This is because exposure of the surface to air leads to an increase of the thermodynamically preferred oxygen adsorption and the replacement of hydroxyl groups adsorbed on the defect sites by oxygen atoms gradually takes place.

Wu et al. synthesized $\mathrm{ZnO}$ structures with three scales of roughness on stainless steel mesh film by a simple hydrothermal method [53]. After being modified with a low surface energy material, for example, Teflon, these films exhibit superhydrophobic and superoleophilic properties. They also demonstrated that the unique properties of the as-prepared films match well with the requirements for the effective separation of oil and water mixtures (Figure 6).

3.3. Chemical Etching. Chemical etching is a process of dissolving metals or semiconductors in acids to make them into a particular shape. It has also attracted many interests in the synthesis of $\mathrm{ZnO}$ superhydrophobic surfaces. Hou et al. fabricated $\mathrm{ZnO}$ submicrorod films (Figure 7) on zinc sheets through an $\mathrm{H}_{2} \mathrm{O}_{2}$-assisted surface etching process and subsequent surface modification with a monolayer of $1 \mathrm{H}, 1 \mathrm{H}, 2 \mathrm{H}, 2 \mathrm{H}$-perfluorodecyltriethoxysilane [63].

3.4. Spray Coating Technique. $\mathrm{ZnO}$ superhydrophobic surfaces have also been fabricated by a simple and cost-effective spray coating technique [64-68]. Wu et al. produced $\mathrm{ZnO}$ superhydrophobic surfaces based on microscale coffee-ring patterns by this technique [64]. The $\mathrm{ZnO}$ crystal cells generated by the degradation of zinc acetate solution at high temperature accumulate at the wetting circle of microdroplet and assemble into a coffee-ring pattern (Figure 8). The diameter of the rings is only about $8 \mu \mathrm{m}$, which is the smallest of any artificial coffee-ring structures in the world. Furthermore, these new patterns are distributed uniformly over the whole macroscale substrate, which is meaningful for real applications. As a result of this finding, the realization of very small biosensors is possible, which means it becomes possible to pack thousands, or even millions, of small microbiosensors onto a single lab-on-a-chip device in a one-step process, 


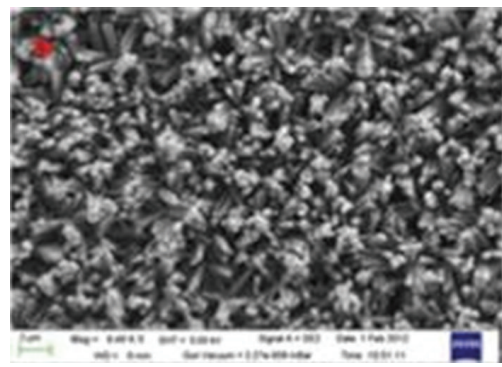

(a)

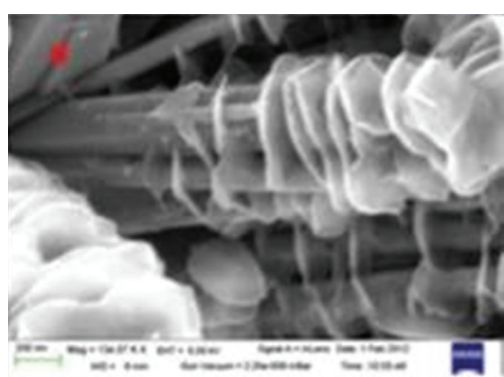

(d)

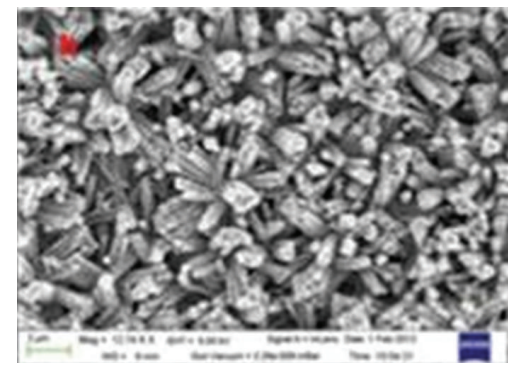

(b)

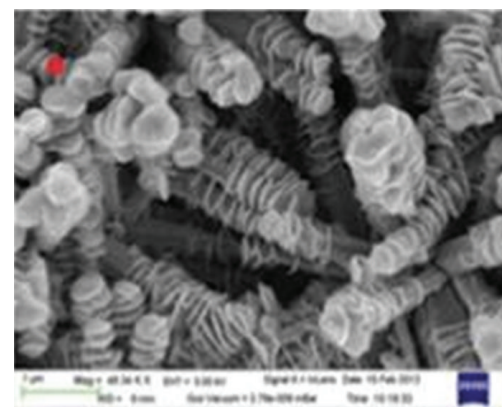

(e)

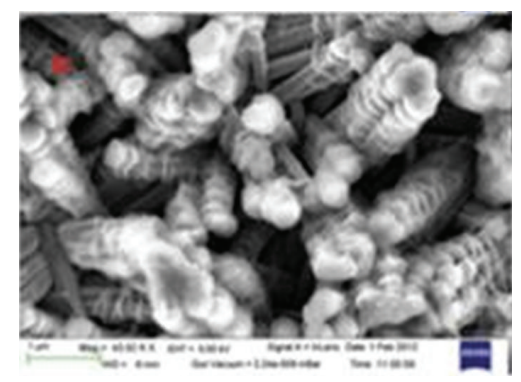

(c)

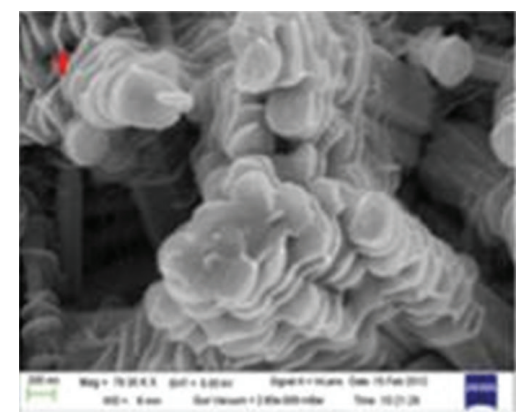

(f)

FIGURE 5: FESEM images of nanocolumnar $\mathrm{ZnO}$; at low magnification ((a), (b)), medium magnification (c); a single nanocolumn at high magnification (d); side view of the nanocolumn (e); tips of the columnar morphology (f). Copyright 2013, RSC.

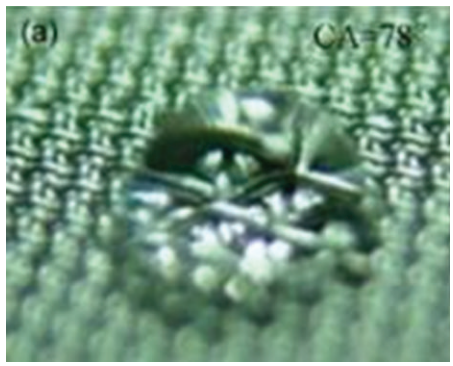

(a)

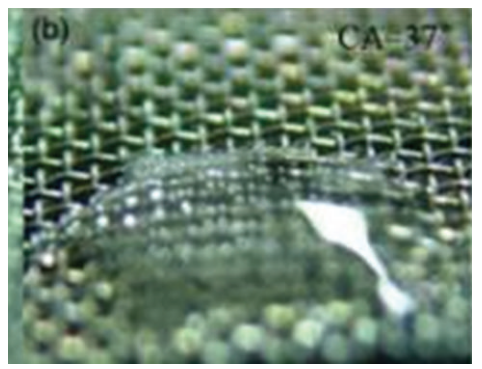

(b)

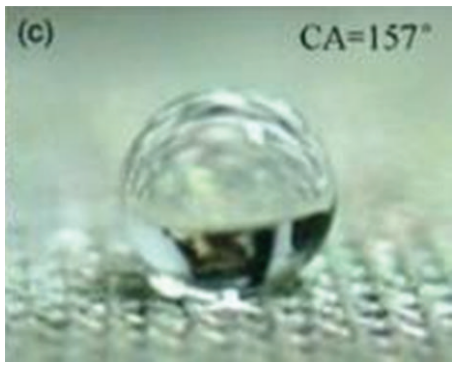

(c)

Figure 6: Water CA investigation: (a) water CA on the original stainless steel mesh; (b) water CA on the mesh with a ZnO hierarchical structure coating; (c) water CA on the as-prepared mesh. Copyright 2012, Wiley.

allowing a large number of medical or chemical analyses to be performed on a single chip.

3.5. Electrochemical Method. Electrochemical method is a way to utilize chemical reactions which take place in a solution at the interface of an electron conductor. These reactions involve electron transfer between the electrode and the electrolyte or species in solution. He et al. fabricated $\mathrm{ZnO}$ thin films with diverse nanostructure, including nanodot, nanowire- and nanoflowers on zinc foils by a simple and rapid electrochemical anodization method [69]. Under the dc or ac electric field, the electroinduced surface wettability conversion from the superhydrophobic to hydrophilic state was observed and the generation of surface defective sites on $\mathrm{ZnO}$ films under electric field was used to explain the transition mechanism. Electroinduced surface wettability conversion is also studied by other researchers [70, 71]. It is found that the reversibility of wettability conversion is dependent on the structure types. This work provides a simple and rapid method for synthesizing different $\mathrm{ZnO}$ nanostructures in large scale, and electric field can be used to modulate the wettability of $\mathrm{ZnO}$ nanostructures.

Hsieh et al. fabricated vertically aligned $\mathrm{ZnO}$ nanorod arrays with different heights on $\mathrm{ZnO}$ seeded indium tin oxide substrate by cathodic electrochemical deposition from zinc nitrate at two temperatures of $60^{\circ} \mathrm{C}$ and $80^{\circ} \mathrm{C}$ [72]. As-grown $\mathrm{ZnO}$ nanorods exhibit wurtzite crystal structure and their heights can be well controlled by different deposition times (Figure 9). The fluorination coating applied on these $\mathrm{ZnO}$ nanorods induces the superhydrophobicity. Actually, as one of the most pronounced morphologies for superhydrophobic applications, $\mathrm{ZnO}$ nanorods/nanowires have been used to change surface characteristics of textiles, polymers, and so forth. The superhydrophobicity of such structure is closely 

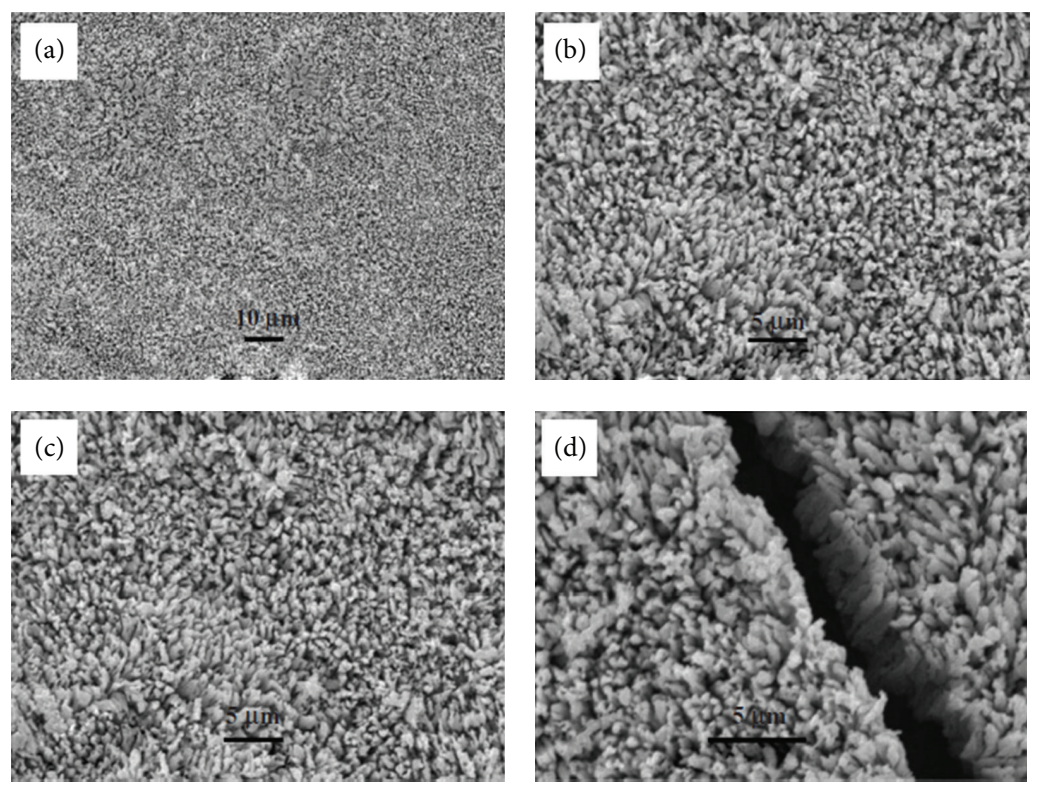

FIGURE 7: SEM images of the prepared $\mathrm{ZnO}$ films on zinc substrate: ((a)-(c)) overview of the $\mathrm{ZnO}$ film surface at various magnifications; (d) overview of the cross section. Copyright 2011, Elsevier.

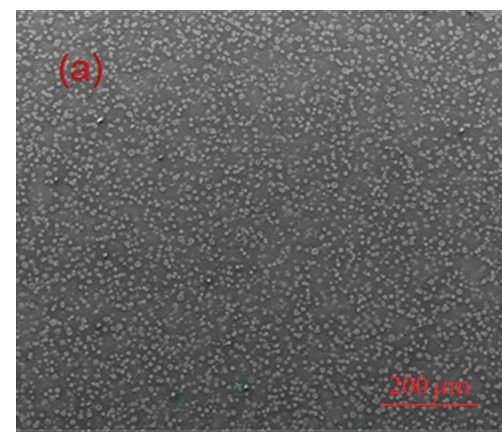

(a)

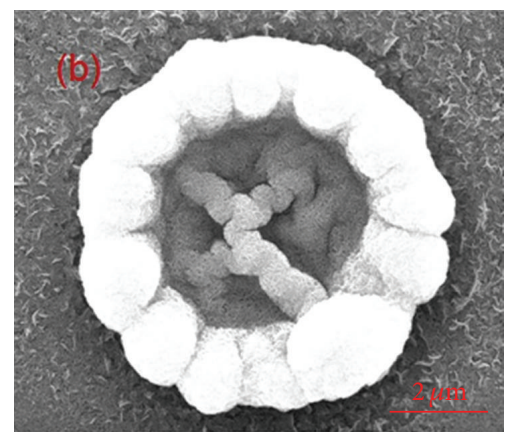

(b)

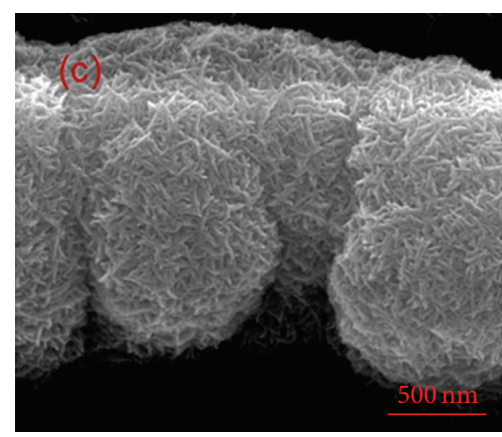

(c)

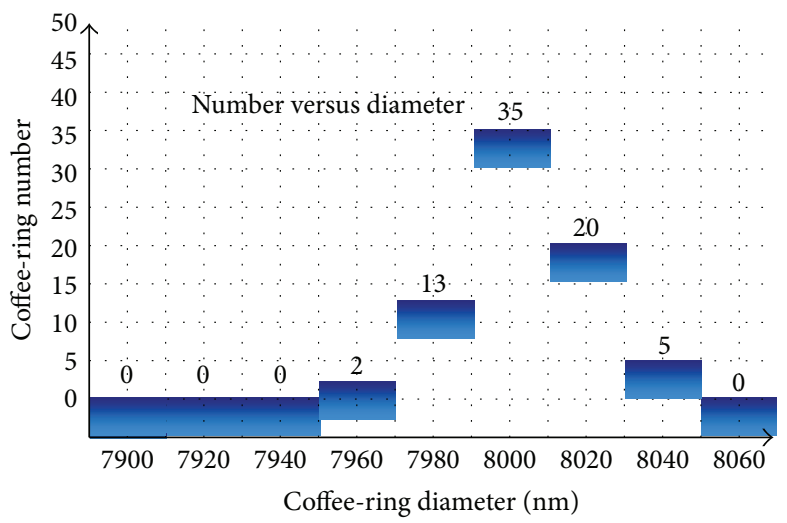

(d)

FIGURE 8: FE-SEM images of the prepared film on a glass substrate at different magnifications. (a) shows a high density array of ring-like structures deposited on the substrate. (b) shows that the diameter of a ring is about $8 \mathrm{~mm}$. (c) is the high-magnification image and shows that the rings surface was covered with a layer of rods. (d) indicates the distribution of the ring diameters. Copyright 2013, RSC. 

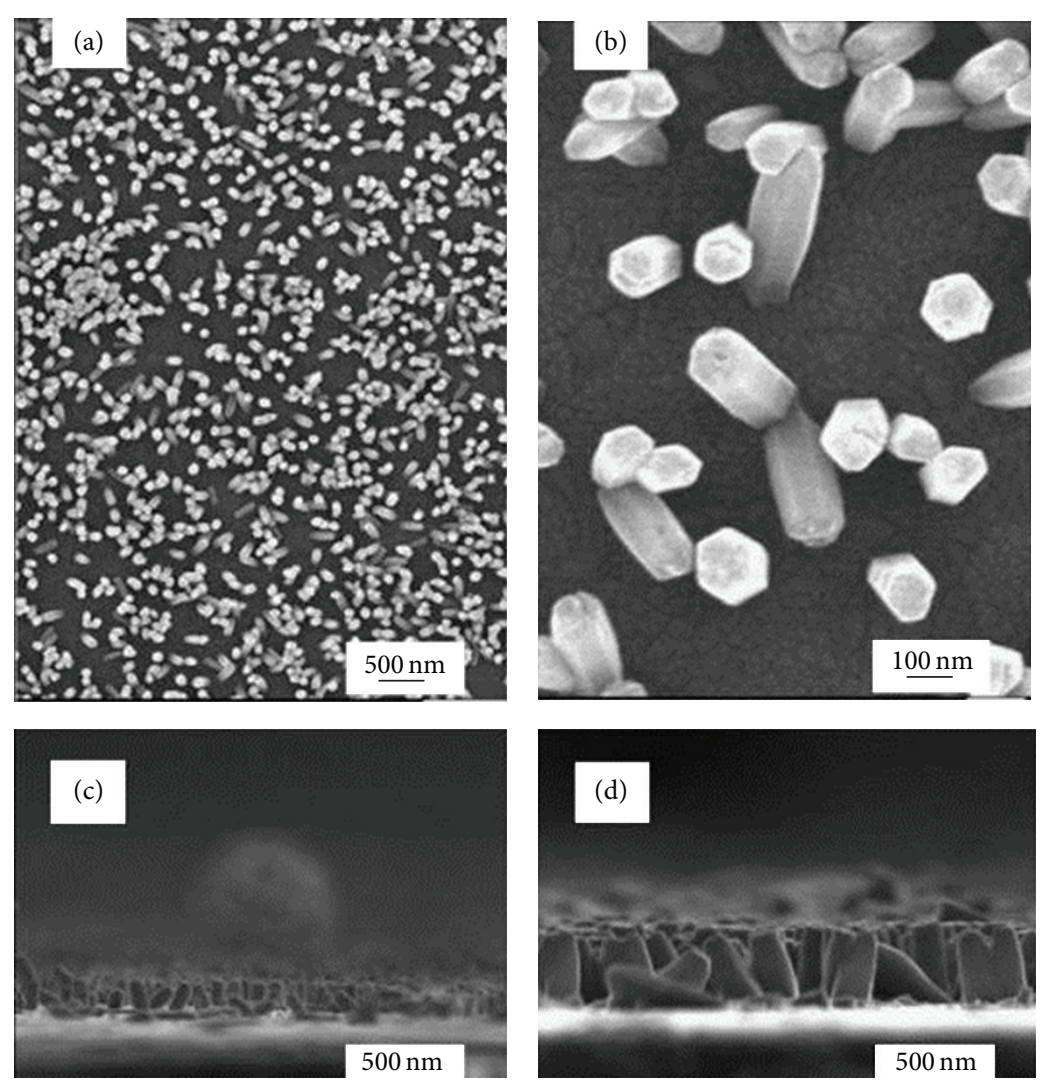

FIGURE 9: Top and cross-sectional views of FE-SEM images of $\mathrm{ZnO}$ nanorods grown onto the $\mathrm{ZnO}$ seed layers by cathodic electrodeposition from $\mathrm{Zn}\left(\mathrm{NO}_{3}\right)_{2}$ at $80^{\circ} \mathrm{C}$ for different periods. Top view: (a) $5 \mathrm{~min}$, (b) $10 \mathrm{~min}$; cross-sectional view: (c) $5 \mathrm{~min}$, (d) $10 \mathrm{~min}$. Copyright 2010, Elsevier.

related to the density of these one-dimensional structures. Briefly, a surface with low density will render the surface with relatively high contact angle.

\section{Conclusions}

In this review, we have tried to represent most of the advances in the domain of $\mathrm{ZnO}$ superhydrophobic surfaces. We focused on the theory advances, synthesis methods, and the multifunctional characteristic of different $\mathrm{ZnO}$ structures. The synthesis approaches reviewed were separated into several types, most of which could realize various surface morphologies with controllable structures. And the related applications benefit from these structures has also been included. This review allows readers to have an easy access to recent advances in this domain in order to find possible approaches to produce such surfaces or to find new functions for interesting applications.

\section{Acknowledgments}

This research was supported by the National Basic Research Program of China (2013CB328803, 2010CB327705), the National High Technology Research and Development Program of China (2012AA03A302, 2013AA011004), National Natural Science Foundation of China (61306140, 51202028),
China Postdoctoral Science Foundation (2013M530222), Natural Science Foundation of Jiangsu Province (BK20130618), and Jiangsu Planned Projects for Postdoctoral Research Funds (1301097C).

\section{References}

[1] P. G. de Gennes, F. Brochard-Wyart, and D. Quere, Capillarity and Wetting Phenomena: Drops, Bubbles, Pearls, Waves, Springer, New York, NY, USA, 2003.

[2] R. Dettre and R. Johnson, "Study of an idealized heterogeneous surface," The Journal of Physical Chemistry, vol. 107, no. 43, pp. 1744-1750, 1963.

[3] A. Solga, Z. Cerman, B. F. Striffler, M. Spaeth, and W. Barthlott, "The dream of staying clean: lotus and biomimetic surfaces," Bioinspiration and Biomimetics, vol. 2, no. 4, pp. S126-S134, 2007.

[4] Y. Zheng, X. Gao, and L. Jiang, "Directional adhesion of superhydrophobic butterfly wings," Soft Matter, vol. 3, no. 2, pp. 178-182, 2007.

[5] G. S. Watson, S. Myhra, B. W. Cribb, and J. A. Watson, "Putative functions and functional efficiency of ordered cuticular nanoarrays on insect wings," Biophysical Journal, vol. 94, no. 8, pp. 3352-3360, 2008.

[6] Y. Liu, X. Chen, and J. H. Xin, "Hydrophobic duck feathers and their simulation on textile substrates for water repellent 
treatment," Bioinspiration and Biomimetics, vol. 3, no. 4, Article ID 046007, 2008.

[7] X. Gao and L. Jiang, "Water-repellent legs of water striders," Nature, vol. 432, no. 7013, p. 36, 2004.

[8] F. Shi, J. Niu, J. Liu et al., "Towards understanding why a superhydrophobic coating is needed by water striders," Advanced Materials, vol. 19, no. 17, pp. 2257-2261, 2007.

[9] D.-G. Lee and H.-Y. Kim, "The role of superhydrophobicity in the adhesion of a floating cylinder," Journal of Fluid Mechanics, vol. 624, pp. 23-32, 2009.

[10] C. W. Wu, X. Q. Kong, and D. Wu, "Micronanostructures of the scales on a mosquito's legs and their role in weight support," Physical Review E, vol. 76, no. 1, Article ID 017301, 4 pages, 2007.

[11] M. R. Flynn and J. W. M. Bush, "Underwater breathing: the mechanics of plastron respiration," Journal of Fluid Mechanics, vol. 608, pp. 275-296, 2008.

[12] A. R. Parker and C. R. Lawrence, "Water capture by a desert beetle," Nature, vol. 414, no. 6859, pp. 33-34, 2001.

[13] L. Zhai, M. C. Berg, F. Ç. Cebeci et al., "Patterned superhydrophobic surfaces: toward a synthetic mimic of the namib desert beetle," Nano Letters, vol. 6, no. 6, pp. 1213-1217, 2006.

[14] X. Feng and L. Jiang, "Design and creation of superwetting/antiwetting surfaces," Advanced Materials, vol. 18, no. 23, pp. 30633078, 2006.

[15] M. Ma and R. M. Hill, "Superhydrophobic surfaces," Current Opinion in Colloid and Interface Science, vol. 11, no. 4, pp. 193202, 2006.

[16] X.-M. Li, D. Reinhoudt, and M. Crego-Calama, "What do we need for a superhydrophobic surface? A review on the recent progress in the preparation of superhydrophobic surfaces," Chemical Society Reviews, vol. 36, no. 8, pp. 1350-1368, 2007.

[17] P. Roach, N. J. Shirtcliffe, and M. I. Newton, "Progess in superhydrophobic surface development," Soft Matter, vol. 4, no. 2, p. 224, 2008.

[18] H. F. Hoefnagels, D. Wu, G. de With, and W. Ming, "Biomimetic superhydrophobic and highly oleophobic cotton textiles," Langmuir, vol. 23, no. 26, pp. 13158-13163, 2007.

[19] F. L. Huang, Q. F. Wei, W. Z. Xu, and Q. Li, "Preparation and characterizations of PTFE gradient nanostructure on silk fabric," Surface Review and Letters, vol. 14, no. 4, pp. 547-551, 2007.

[20] X. Zhang, M. Jin, Z. Liu et al., "Superhydrophobic $\mathrm{TiO}_{2}$ surfaces: preparation, photocatalytic wettability conversion, and superhydrophobic-superhydrophilic patterning," The Journal of Physical Chemistry C, vol. 111, no. 39, pp. 14521-14529, 2007.

[21] N. D. Boscher, P. Choquet, D. Duday, and S. Verdier, "Chemical compositions of organosilicon thin films deposited on aluminium foil by atmospheric pressure dielectric barrier discharge and their electrochemical behaviour," Surface and Coatings Technology, vol. 205, no. 7, pp. 2438-2448, 2010.

[22] E. Hosono, S. Fujihara, I. Honma, and H. Zhou, "Superhydrophobic perpendicular nanopin film by the bottom-up process," Journal of the American Chemical Society, vol. 127, no. 39, pp. 13458-13459, 2005.

[23] X. Zhang, F. Shi, X. Yu et al., "Fabrication of superhydrophobic surface from a supramolecular organosilane with quadruple hydrogen bonding," Journal of the American Chemical Society, vol. 126, no. 15, pp. 4796-4797, 2004.

[24] M. E. Buck, S. C. Schwartz, and D. M. Lynn, "Superhydrophobic thin films fabricated by reactive layer-by-layer assembly of azlactone-functionalized polymers," Chemistry of Materials, vol. 22, no. 23, pp. 6319-6327, 2010.
[25] Y. Ofir, B. Samanta, P. Arumugam, and V. M. Rotello, “Controlled fluorination of FePt nanoparticles: hydrophobic to superhydrophobic surfaces," Advanced Materials, vol. 19, no. 22, pp. 4075-4079, 2007.

[26] K. Ramaratnam, V. Tsyalkovsky, V. Klep, and I. Luzinov, "Ultrahydrophobic textile surface via decorating fibers with monolayer of reactive nanoparticles and non-fluorinated polymer," Chemical Communications, no. 43, pp. 4510-4512, 2007.

[27] L. Zhai, F. C. Cebeci, R. E. Cohen, and M. F. Rubner, "Stable superhydrophobic coatings from polyelectrolyte multilayers," Nano Letters, vol. 4, no. 7, pp. 1349-1353, 2004.

[28] X. Zhang, H. Kono, Z. Liu et al., "A transparent and photo-patternable superhydrophobic film," Chemical Communications, no. 46, pp. 4949-4951, 2007.

[29] T. J. Wood, G. A. Hurst, W. C. E. Schofield et al., "Electroless deposition of multi-functional zinc oxide surfaces displaying photoconductive, superhydrophobic, photowetting, and antibacterial properties," Journal of Materials Chemistry, vol. 22, no. 9, pp. 3859-3867, 2012.

[30] I. Yilgor, S. Bilgin, M. Isik, and E. Yilgor, "Tunable wetting of polymer surfaces," Langmuir, vol. 28, no. 41, pp. 14808-14814, 2012.

[31] G.-Y. Li, X.-P. Li, H. Wang, Z.-Q. Yang, J.-Y. Yao, and G.-F. Ding, "Fabrication and characterization of superhydrophobic surface by electroplating regular rough micro-structures of metal nickel," Microelectronic Engineering, vol. 95, pp. 130-134, 2012.

[32] F. Li, X. Geng, Z. Chen, and L. Zhao, "A novel and expeditious method to fabricate superhydrophobic metal carboxylate surface," Applied Physics A, vol. 106, no. 1, pp. 35-40, 2012.

[33] Y. Park, M. Han, and Y. Ahn, "Fabrication of superhydrophobic metal surfaces with self-assembled monolayers of silane derivatives having inter-hydrogen bonding," Bulletin of the Korean Chemical Society, vol. 32, no. 3, pp. 1091-1094, 2011.

[34] D. Ebert and B. Bhushan, "Transparent, superhydrophobic, and wear-resistant coatings on glass and polymer substrates using $\mathrm{SiO}_{2}, \mathrm{ZnO}$, and ITO nanoparticles," Langmuir, vol. 28, no. 31, pp. 11391-11399, 2012.

[35] P. Escalé, L. Rubatat, L. Billon, and M. Save, "Recent advances in honeycomb-structured porous polymer films prepared via breath figures," European Polymer Journal, vol. 48, no. 6, pp. 1001-1025, 2012.

[36] N. J. Shirtcliffe, G. McHale, and M. I. Newton, "The superhydrophobicity of polymer surfaces: recent developments," Journal of Polymer Science B, vol. 49, no. 17, pp. 1203-1217, 2011.

[37] Y.-L. Zhang, J.-N. Wang, Y. He et al., "Solvothermal synthesis of nanoporous polymer chalk for painting superhydrophobic surfaces," Langmuir, vol. 27, no. 20, pp. 12585-12590, 2011.

[38] D. Nystrom, P. Antoni, S. Holdcroft, A. Hult, E. M. Jonsson, and G. Vamvounis, "Solution-processed superhydrophobic conjugated polymer films," Soft Matter, vol. 8, no. 21, pp. 5753-5755, 2012.

[39] P. Atienzar, T. Ishwara, B. N. Illy et al., "Control of photocurrent generation in polymer/ZnO nanorod solar cells by using a solution-processed $\mathrm{TiO}_{2}$ overlayer," The Journal of Physical Chemistry Letters, vol. 1, no. 4, pp. 708-713, 2010.

[40] R. Könenkamp, R. C. Word, and C. Schlegel, "Vertical nanowire light-emitting diode," Applied Physics Letters, vol. 85, no. 24, pp. 6004-6006, 2004.

[41] Z. H. Lim, Z. X. Chia, M. Kevin, A. S. W. Wong, and G. W. Ho, "A facile approach towards $\mathrm{ZnO}$ nanorods conductive textile for room temperature multifunctional sensors," Sensors and Actuators $B$, vol. 151, no. 1, pp. 121-126, 2010. 
[42] C. Soci, A. Zhang, B. Xiang et al., "ZnO nanowire UV photodetectors with high internal gain," Nano Letters, vol. 7, no. 4, pp. 1003-1009, 2007.

[43] J. Wu, J. Xia, W. Lei, and B. Wang, "Facile synthesis of threedimensional zno nanostructure: realization of a multifunctional stable superhydrophobic surface," PLoS ONE, vol. 6, no. 12, Article ID e29047, 2011.

[44] J. Liang, Y. Hu, Y. Wu, and H. Chen, "Fabrication and corrosion resistance of superhydrophobic hydroxide zinc carbonate film on aluminum substrates," Journal of Nanomaterials, vol. 2013, Article ID 139768, 6 pages, 2013.

[45] Q. Li, Y. Q. Chen, L. B. Luo, L. Wang, Y. Q. Yu, and L. Zhai, "Photoluminescence and wetting behavior of $\mathrm{ZnO}$ nanoparticles/nanorods array synthesized by thermal evaporation," Journal of Alloys and Compounds, vol. 560, pp. 156-160, 2013.

[46] D. Quéré, "Non-sticking drops," Reports on Progress in Physics, vol. 68, no. 11, pp. 2495-2532, 2005.

[47] R. N. Wenzel, "Resistance of solid surfaces to wetting by water," Industrial \& Engineering Chemistry Research, vol. 28, pp. 988994, 1936.

[48] A. B. D. Cassie and S. Baxter, "Wettability of porous surfaces," Transactions of the Faraday Society, vol. 40, pp. 546-551, 1944.

[49] V. E. B. Dussan and R. T.-P. Chow, "On the ability of drops or bubbles to stick to non-horizontal surfaces of solids," Journal of Fluid Mechanics, vol. 137, pp. 1-29, 1983.

[50] H. C. Barshilia, S. John, and V. Mahajan, "Nanometric multiscale rough, transparent and anti-reflective $\mathrm{ZnO}$ superhydrophobic coatings on high temperature solar absorber surfaces," Solar Energy Materials and Solar Cells, vol. 107, pp. 219-224, 2012.

[51] U. P. Shaik, S. Kshirsagar, M. G. Krishna, S. P. Tewari, D. Dhar Purkayastha, and V. Madhurima, "Growth of superhydrophobic zinc oxide nanowire thin films," Materials Letters, vol. 75, pp. 5153, 2012.

[52] C. Mondal, M. Ganguly, A. K. Sinha, J. Pal, and T. Pal, "Fabrication of a $\mathrm{ZnO}$ nanocolumnar thin film on a glass slide and its reversible switching from a superhydrophobic to a superhydrophilic state," RSC Advances, vol. 3, pp. 5937-5944, 2013.

[53] J. Wu, J. Chen, K. Qasim, J. Xia, W. Lei, and B.-P. Wang, "A hierarchical mesh film with superhydrophobic and superoleophilic properties for oil and water separation," Journal of Chemical Technology and Biotechnology, vol. 87, no. 3, pp. 427-430, 2012.

[54] E. S. Ates and H. E. Unalan, "Zinc oxide nanowire enhanced multifunctional coatings for cotton fabrics," Thin Solid Films, vol. 520, no. 14, pp. 4658-4661, 2012.

[55] P. Pannasri, P. Siriphannon, P. Monvisade, and J. Nookaew, "Hydrothermal growth of $\mathrm{ZnO}$ nanostructures from nano- $\mathrm{ZnO}$ seeded in P(MMA-co-BA) matrix," Journal of Polymer Research, vol. 18, no. 6, pp. 2245-2254, 2011.

[56] M. T. Z. Myint, R. Kitsomboonloha, S. Baruah, and J. Dutta, "Superhydrophobic surfaces using selected zinc oxide microrod growth on ink-jetted patterns," Journal of Colloid and Interface Science, vol. 354, no. 2, pp. 810-815, 2011.

[57] A. K. Sinha, M. Basu, M. Pradhan, S. Sarkar, and T. Pal, "Fabrication of large-scale hierarchical $\mathrm{ZnO}$ hollow spheroids for hydrophobicity and photocatalysis," Chemistry, vol. 16, no. 26, pp. 7865-7874, 2010.

[58] Y. Sun, R. Zou, W. Li et al., "A controllable hydrothermal synthesis of uniform three-dimensional hierarchical microstructured ZnO films," CrystEngComm, vol. 13, no. 20, pp. 6107-6113, 2011.
[59] R. Dufour, G. Perry, M. Harnois et al., "From micro to nano reentrant structures: hysteresis on superomniphobic surfaces," Colloid \& Polymer Science, vol. 291, pp. 409-415, 2013.

[60] J. Wu, J. Xia, W. Lei, and B. Wang, "Superhydrophobic surface based on a coral-like hierarchical structure of ZnO," PLoS ONE, vol. 5, no. 12, Article ID e14475, 2010.

[61] M. T. Z. Myint, N. S. Kumar, G. L. Hornyak, and J. Dutta, "Hydrophobic/hydrophilic switching on zinc oxide microtextured surface," Applied Surface Science, vol. 264, pp. 344-348, 2013.

[62] J. Wu, J. Xia, W. Lei, and B.-P. Wang, "A one-step method to fabricate lotus leaves-like $\mathrm{ZnO}$ film," Materials Letters, vol. 65, no. 3, pp. 477-479, 2011.

[63] X. Hou, L. Wang, F. Zhou, and L. Li, "Fabrication of $\mathrm{ZnO}$ submicrorod films with water repellency by surface etching and hydrophobic modification," Thin Solid Films, vol. 519, no. 22, pp. 7813-7816, 2011.

[64] J. Wu, J. Xia, W. Lei, and B. P. Wang, "Generation of the smallest coffee-ring structures by solute crystallization reaction on a hydrophobic surface," RSC Advances, vol. 3, no. 16, pp. 53285331, 2013.

[65] N. L. Tarwal and P. S. Patil, "Superhydrophobic and transparent $\mathrm{ZnO}$ thin films synthesized by spray pyrolysis technique," Applied Surface Science, vol. 256, no. 24, pp. 7451-7456, 2010.

[66] N. L. Tarwal, V. M. Khot, N. S. Harale et al., "Spray deposited superhydrophobic $\mathrm{ZnO}$ coatings via seed assisted growth," Surface and Coatings Technology, vol. 206, no. 6, pp. 1336-1341, 2011.

[67] A. Steele, I. Bayer, S. Moran, A. Cannon, W. P. King, and E. Loth, "Conformal $\mathrm{ZnO}$ nanocomposite coatings on micro-patterned surfaces for superhydrophobicity," Thin Solid Films, vol. 518, no. 19, pp. 5426-5431, 2010.

[68] J. Wu, J. Xia, Y.-N. Zhang, W. Lei, and B.-P. Wang, "A simple method to fabricate the different extents of superhydrophobic surfaces," Physica E, vol. 42, no. 5, pp. 1325-1328, 2010.

[69] S. He, M. Zheng, L. Yao et al., "Preparation and properties of $\mathrm{ZnO}$ nanostructures by electrochemical anodization method," Applied Surface Science, vol. 256, no. 8, pp. 2557-2562, 2010.

[70] J. Wu, J. Xia, J. Chen, W. Lei, and B. P. Wang, "Wetting transition on Micro/Nanostructured ZnO layers," Journal of Adhesion Science and Technology, vol. 26, no. 12-17, pp. 2099-2104, 2012.

[71] J. Wu, J. Xia, W. Lei, and B.-P. Wang, "Electrowetting on $\mathrm{ZnO}$ nanowires," Applied Physics A, vol. 99, no. 4, pp. 931-934, 2010.

[72] C.-T. Hsieh, S.-Y. Yang, and J.-Y. Lin, "Electrochemical deposition and superhydrophobic behavior of $\mathrm{ZnO}$ nanorod arrays," Thin Solid Films, vol. 518, no. 17, pp. 4884-4889, 2010.

[73] M. Zhou, J. Li, C.-C. Feng, C.-X. Wu, R. Yuan, and L. Cai, "Bionic superhydrophobic and drag-reduction properties on ZnO-nanostructured functional surfaces," Chemical Vapor Deposition, vol. 16, no. 1-3, pp. 12-14, 2010.

[74] M. Ashraf, C. Campagne, A. Perwuelz, P. Champagne, A. Leriche, and C. Courtois, "Development of superhydrophilic and superhydrophobic polyester fabric by growing zinc Oxide nanorods," Journal of Colloid and Interface Science, vol. 394, pp. 545-553, 2013.

[75] N. Preda, M. Enculescu, I. Zgura et al., "Superhydrophobic properties of cotton fabrics functionalized with $\mathrm{ZnO}$ by electroless deposition," Materials Chemistry and Physics, vol. 138, pp. 253-261, 2013.

[76] Y. Wan, Z. Wang, Y. Liu, C. Qi, and J. Zhang, "Reducing friction and wear of a zinc substrate by combining a stearic acid overcoat 
with a nanostructured zinc oxide underlying film: perspectives to super-hydrophobicity," Tribology Letters, vol. 44, no. 3, pp. 329-333, 2011.

[77] J. Wu, J. Xia, W. Lei, and B.-P. Wang, "Fabrication of superhydrophobic surfaces with double-scale roughness," Materials Letters, vol. 64, no. 11, pp. 1251-1253, 2010.

[78] S. Lu, Y. Chen, W. Xu, and W. Liu, "Controlled growth of superhydrophobic films by sol-gel method on aluminum substrate," Applied Surface Science, vol. 256, no. 20, pp. 6072-6075, 2010. 

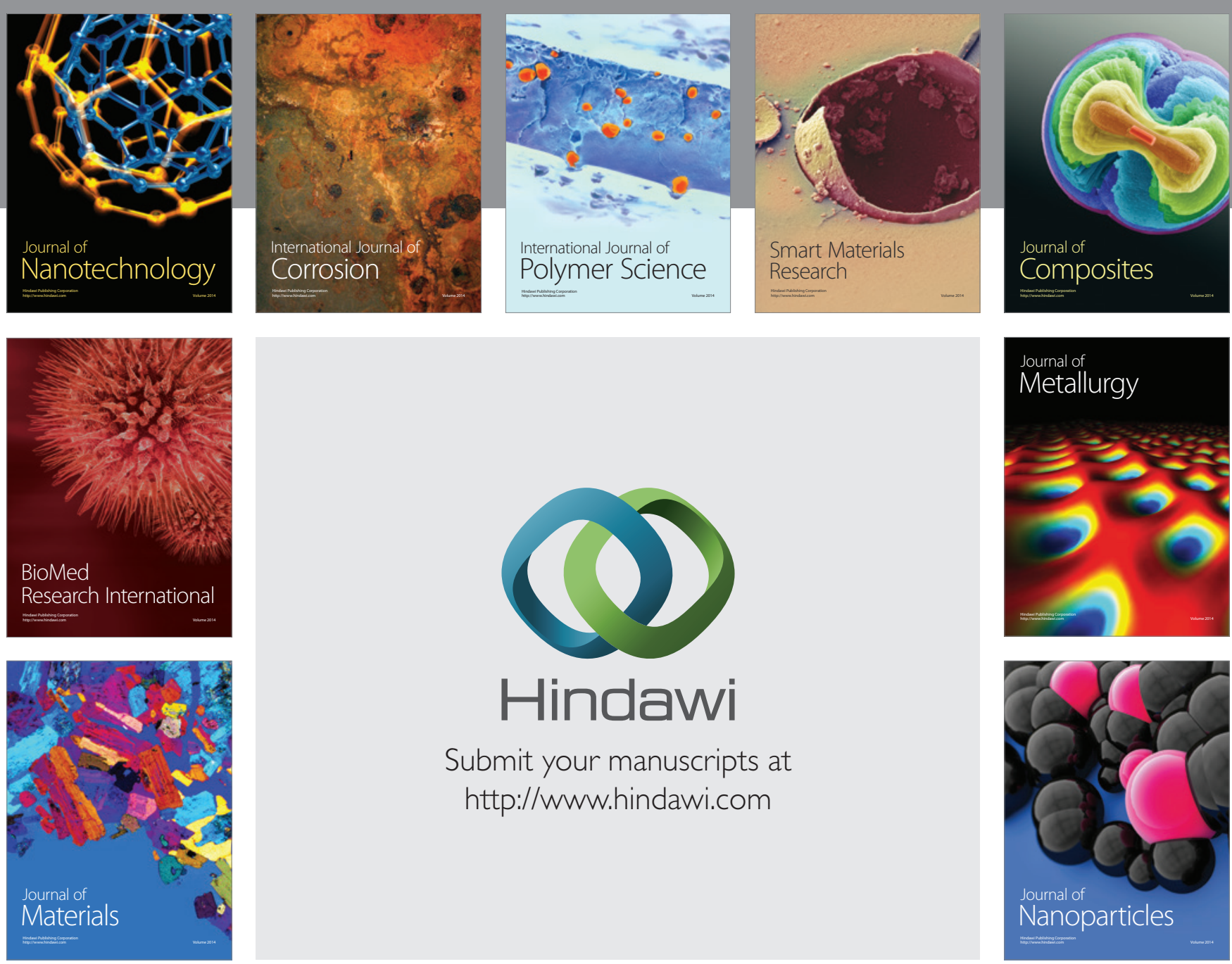

Submit your manuscripts at http://www.hindawi.com
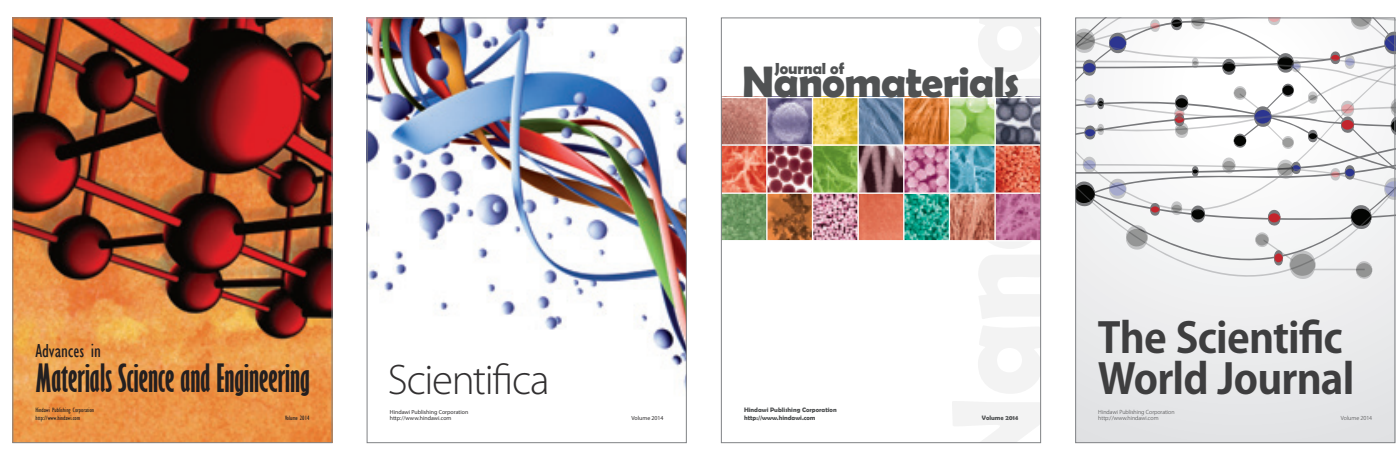

\section{The Scientific World Journal}
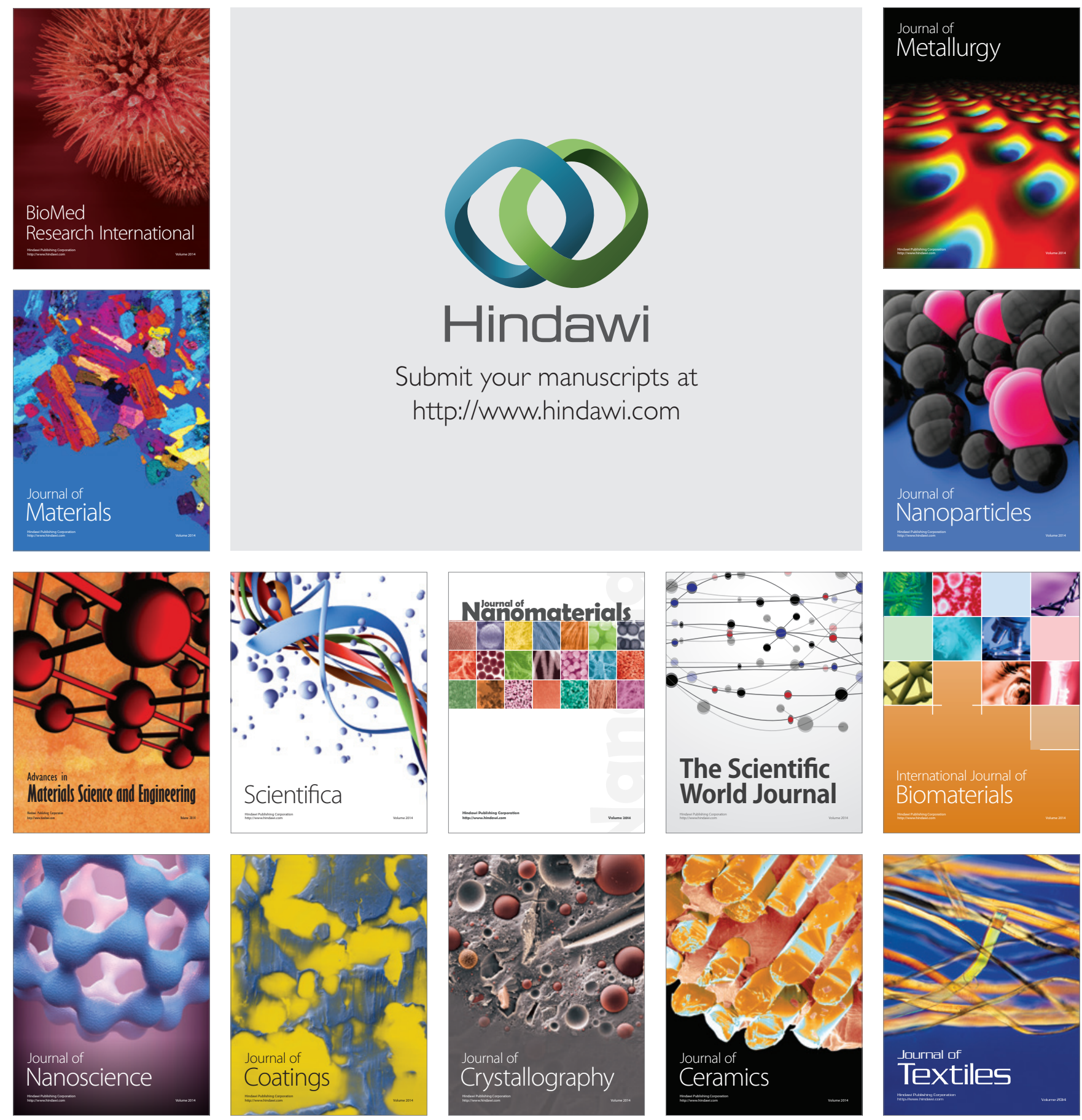